ge im weiteren Trainingsregime führten zur vorzeitigen Beendigung mancher hoffnungsvoller Sportlerkarriere, weshalb auch dem Wissen der Instrumentallehrer um die funktionellen Grundlagen des Kiefergelenkes, seiner Muskulatur und anderer Faktoren der instrumentellen Leistungsfähigkeit bei Bläsern und Sängern nicht zuviel Vorschusslorbeer zugestanden werden darf.

Können wir analog zu den verschiedenen Sportdisziplinen Elemente einer Leistungsstruktur des Bläsers oder des Sängers beschreiben, die wenigstens die

Manuelle Medizin 2006 · 44:345-346

DOI 10.1007/s00337-006-0454-0

Online publiziert: 4. August 2006

(c) Springer Medizin Verlag 2006
Funktionen des Kiefergelenkes, der mimischen, supra- und infrahyoidalen Muskulatur einschließen?

Übereinstimmend mit den Autoren und Frau Steinmetz will ich abschließend verdeutlichen, dass viele ernsthafte Untersuchungen und Überlegungen notwendig sein werden, ehe die funktionsmedizinische Betreuung und Entwicklung von Musikern im Struktur- und Funktionselement Stütz- und Bewegungssystem mit dem Schwerpunkt Kiefergelenk und dessen weit zu fassender Funktionsmuskulatur auf ein professionelles medizinisches
Niveau nach manualmedizinischem Funktionsverständnis gebracht ist.

\section{Korrespondierender Autor}

\section{Dr. B. Krocker}

Gemeinschaftspraxis

Dresdener Straße 18, 03050 Cottbus

DrBK@gmx.net

\section{Literatur}

1. Strauzenberg et al. (Hrsg) (1990) Sportmedizin. Barth, Leipzig

\title{
Asymmetrien der okzipitozervikalen Region
}

Zum Beitrag

Koch LE, Korbmacher H (2006)

Röntgenmorphologie der okzipitozervikalen Region in Abhängigkeit vom Alter Manuelle Medizin 44: 117-120

\section{Leserbrief}

\section{A. Cramer}

Ibbenbüren

Zur Seitenpräferenz im atlantookzipitalen Bereich machte schon Jirout in mehreren seiner Veröffentlichungen -auch in $\mathrm{Ma}$ nuelle Medizin - statistisch gut belegte Angaben, deren Quintessenz sich mit denen der oben genannten Autoren weitgehend decken.

Es ist in der Tat von Belang, bei manueller Therapie im Genick die vorherrschende Asymmetrie zu beachten, die wie von den Autoren bemerkt - altersabhängig ist.

In Übereinstimmung mit Jirout nimmt nach meiner Beobachtung die Linksprävalenz der Asymmetrie, und damit die Linksprävalenz der Störung, ins Senium hin- ein zu. Jenseits des etwa 6o. Lebensjahres kann manuelle Therapie im Subokzipitalbereich funktionell von einer Verschiebung der Linksrotation ausgehen.

Weil aber Röntgenbilder gewöhnlich am ruhenden (meist liegenden) Patienten aufgenommen werden, bleibt der Einfluss der statischen Balance aus dem Iliosakralbereich, den Hüftgelenken und den Beinen außer Betracht.

Die Wirbelsäule muss als funktionelle Einheit aufgefasst werden, in der sowohl Balancefehler und Asymmetrien vom Becken her bis in die Kopfgelenke durchgeschoben werden (dazu die Lowett-Regeln) als auch Balancefehler und Asymmetrien aus den Kopfgelenken die Symmetrie hinab in den Lenden-Becken-HüftBereich bis in die Füße beeinflussen.

Es hat sich für uns als nützlich erwiesen, vor manueller Therapie den Patienten unbekleidet stehend vor einer waagerechten Markierung (z. B. Fensterkreuz, Türfüllung, Tischkante) zu betrachten. Dabei wird „das hängende Ohr“ und der „hängende Beckenkamm“ ebenso registriert wie die „Abweichung der Laufrichtung von der Rumpfsagittalen“ (Füße in
"Schlussstellung“) und die Abweichung der Blickrichtung (Kopfhaltung) von der Rumpfsagittalen und Laufrichtung.

Damit erfassen wir die Asymmetrien der lotrechten und sagittalen Körperachse, die miteinander in funktionellem und neurokoordinatorischem Kontext verstanden sein wollen.

Die von den Autoren aufgeworfene Frage nach der Bedeutung der „Asymmetrie“ im Menschenbild der manuellen Therapie ist von sehr grundsätzlicher Bedeutung.

Meiner Meinung nach liefert nicht Kepplers „Harmonie des Kosmos“ das wahre Bild von der Evolution, der auch wir unterworfen sind, sondern vielmehr jene diskrete Asymmetrie, der wir bei genauerem Hinsehen im Großen wie im Kleinen begegnen.

Darum sollte der Manualtherapeut dem Patienten „seine“ Asymmetrie lassen.

\section{Korrespondierender Autor}

Dr. A. Cramer

(Mitbegründer der FAC)

Bäumerstraße 12, 49477 Ibbenbüren 